\title{
COMPARATIVE STUDY OF SPINAL AND LOCAL ANESTHESIA WITH PROPOFOL INFUSION FOR KNEE ARTHROSCOPY
}

Robson Rocha da Silva', Marcos Almeida Matos², Gleise Madureira ${ }^{3}$, Indiara Gouveia dos Santos ${ }^{4}$

\section{ABSTACT}

Spinal anesthesia for knee arthroscopy is a well-documented and safe procedure. However, some complications and higher costs have been reported. Also, many orthopaedic surgeons are reluctant to use local anesthesia for fear of having to convert to general anesthesia due to inadequate pain control. The purpose of this study is to compare local with spinal anesthesia in two groups of patients submitted to knee arthroscopy. Sixty-five patients were divided in two groups; based on the anesthesia method used, and submitted to the same surgical routine and postoperative analgesia protocol. They were evaluated for analgesia, level of postoperative pain, and level of satisfaction with the type of anesthetic. The two groups did not present any significant differences in relation to perioperative analgesia and pain on the first postoperative day, neither was there

\section{INTRODUCTION}

Knee arthroscopy is an approved medical procedure of importance in diagnosing and treating a variety of pathological conditions. Since its beginnings, with Takagi, Bircher and Watanabe, it has undergone extraordinary evolution, thanks to improvements in the arthroscopic technique and technological advances. According to Jackson ${ }^{(1)}$, the advent of arthroscopy was one of the most important events within orthopedics during the $20^{\text {th }}$ century, since it introduced the concept of minimally invasive surgery.

It is traditionally performed under spinal block anesthesia and contraindications are rare. Moreover, knee arthroscopy presents a low complication rate. Nonetheless, in some situations, there are any difference in relation to emotional state. However, there was a significant difference in terms of acceptance of the procedure; $100 \%$ said they would accept the procedure again in the local anesthesia group, compared with $60.5 \%$ in the spinal anesthesia group; also, $100 \%$ in the local anesthesia group said they felt encouraged by the type of anesthesia, compared with $67.7 \%$ in the spinal anesthesia group. We can conclude that local anesthesia is similar to spinal anesthesia in almost all the aspects investigated, except in terms of acceptance and patients' level of satisfaction with the procedure. Local anesthesia can be a good alternative to spinal anesthesia, especially in outpatient departments, or when patients have restrictions to traditional models of anesthesia.

Keywords - Arthroscopy; Knee; Anesthesia

\footnotetext{
1- Orthopedic Physician, Head of the Knee Group of the Hospital Santa Izabel da Santa Casa de Misericórdia da Bahia - Salvador, BA, Brazil.

2- Tensurd Professor and Doctor of the Escola Baiana de Medicina e Saúde Pública - Salvador, BA, Brazil.

3-Anesthesist of the Hospital Santa Izabel da Santa Casa de Misericórdia da Bahia - Salvador, BA, Brazil.

4- Resident Physician of the Hospital Santa Izabel da Santa Casa de Misericórdia da Bahia - Salvador, BA, Brazil.

Work carried out at the Hospital Santa Izabel da Santa Casa de Misericórdia da Bahia - Salvador, BA, Brazil.

Correspondence: Av. Orlando Gomes, Condomínio Veredas Piatã, Quadra B Lote 07, Bairro - Piatã - Salvador, BA - 41650-010 - E-mail robroc@superig.com.br Received for publication: 11/15/2010, accepted for publication: 05/25/2011.
}

The authors declare that there was no conflict of interest in conducting this work 


\section{Sample and Methods}

The study population was formed by patients registered at the Knee Surgery Service of Hospital Santa Izabel da Santa Casa de Misericórdia da Bahia. Patients were included between April 5, 2003, and August 28, 2006. They were over the age of 15 years, of both sexes, with a medical indication for knee arthroscopy and a diagnosis of traumatic or degenerative meniscal lesion. They were all operated with the simultaneous presence of the same surgeon and the anesthetist (the authors of this study). Patients presenting low levels of cognition, ligament lesions or associated fractures were excluded.

The sample consisted of sequential selection of 65 patients who were divided consecutively and randomly into one group or the other. This sample represented $8.12 \%$ of the patients operated at this service over the study period and $16.25 \%$ of the patients operated by the author.

Thirty-two of these patients were operated under spinal block anesthesia and were included in the block group (BL). The other 33 patients were operated under local anesthesia and sedation with Propofol, and were included in the local anesthesia group (LA).

The ages in the BL group ranged from 20 to 71 years (mean of $41 \pm 13.16$ ); 15 patients were male and 17 were female. The ages in the LA group ranged from 15 to 72 years (mean of $50 \pm 13.36$ ); 16 patients were male and 17 were female.

\section{Anesthetic procedure}

All the patients were informed in advance about the type of anesthesia that would be used.

In the BL group, the anesthetist made a puncture in the L4-L5 intervertebral space, and $10 \mathrm{mg}$ of $0.5 \%$ isobaric Marcaine ${ }^{\circledR}$ was infused. All the patients firstly received pre-anesthesia consisting of $50 \mathrm{mcg}$ of fentanyl and $1 \mathrm{mg}$ of midazolam.

In the LA group, the anesthetist made a peripheral venous puncture and sedation was administered consisting of $1 \%$ propofol in a target infusion controlled according to medical criteria. If the patients presented anxiety, $0.05 \mathrm{mcg} / \mathrm{kg} / \mathrm{min}$ of remifentanil was administered. After implementing asepsis and antisepsis and setting up the operating fields, the surgeon administered an infiltration of $5 \mathrm{ml}$ of $2 \%$ lidocaine through the portals and, just afterwards, intra-articular infiltration of $20 \mathrm{ml}$ of $0.5 \%$ bupivacaine with a vasoconstrictor.

After completing the anesthetic procedure in the two groups, the limb was elevated for 5 minutes and a pneumatic tourniquet was applied. At the end of the surgery, $2 \mathrm{~g}$ of dipyrone and $30 \mathrm{mg}$ de ketorolac were administered.

All the patients underwent the same surgical routine and the same postoperative analgesia protocol, consisting of $100 \mathrm{mg}$ ketoprofen orally every 12 hours, in association (when necessary) with $30 \mathrm{mg}$ of codeine and $500 \mathrm{mg}$ of paracetamol orally, every 6 hours. The patients were always discharged from hospital on the first postoperative day in both groups.

At the time of hospital discharge, when completely lucid and aware of their surroundings, the patients were invited to answer the study questionnaire, which consisted of subjective assessment of their pain, the acceptability of the method, their emotional state and the influence of the type of anesthesia on their acceptance of the surgery.

The patients' perception of pain was assessed on relation to two different times: 1) pain during the operation, assessed by asking them about any memory of pain or discomfort felt on the previous day at the time of the surgery, with two possible responses (yes or no); 2) postoperative pain, assessed by asking them to rate the pain they felt at that moment, using a visual analogue scale (VAS).

The patients were asked about their emotional state just before the surgery, when they learned which type of anesthesia would be used. They were instructed to mark one of three options: calm; slightly nervous; or very nervous.

The data are presented in tables of frequency distribution. Comparisons between the groups were made using the chi-square or Fisher exact test when appropriate (groups with fewer than five samples) and the significance level used was 0.05 .

\section{Ethical issues}

The study protocol was approved by the Research Ethics Committee of Santa Casa de Misericórdia da Bahia, and all the patients were given prior explanations about the research. All patients who freely agreed to participate in the study signed a free and informed consent statement.

\section{RESULTS}

The two groups were homogenous regarding gender. The percentage of men was $46.87 \%$ in the BL group and $48.48 \%$ in the LA group. The groups were also homogenous regarding age. 
The type of anesthesia used for the two groups was satisfactory for carrying out the proposed procedure. Hospital discharge was given in all cases on the first postoperative day.

With regard to pain during the surgery (Table 1), only one patient in the BL group (3.12\%) reported feeling pain during the procedure. In the LA group, two patients $(6.06 \%)$ reported feeling pain during the surgery, but the pain did not prevent the procedure from being performed, and it would not dissuade the patients from undergoing new surgery with the same anesthesia.

The patients in both groups presented pain scores between 0 and 5 , without any statistically significant difference between the groups. With regard to postoperative pain, the data are shown in Table 2.

The patients' emotional state before the surgery, encouragement to undergo the procedure and acceptance of a new intervention under the same anesthesia are described in the data presented in Tables 3, 4 and 5 , respectively.

Table 1 - Reported pain during the surgery.

\begin{tabular}{c|c|c}
\hline & Yes & No \\
\hline Block & $1(3.125 \%)$ & $31(96.875 \%)$ \\
\hline Analgesia & $2(6.06 \%)$ & $31(93.93 \%)$ \\
\hline$P=0.978$.
\end{tabular}

Table 2 - Pain distribution on the first postoperative day, according to VAS.

\begin{tabular}{c|c|c|c|c|c|c}
\hline & 0 & 1 & 2 & 3 & 4 & 5 \\
\hline Block & $\begin{array}{c}18 \\
(56.25 \%)\end{array}$ & $\begin{array}{c}6 \\
(18.75 \%)\end{array}$ & $\begin{array}{c}3 \\
(9.37 \%)\end{array}$ & $\begin{array}{c}3 \\
(9.37 \%)\end{array}$ & $\begin{array}{c}1 \\
(3.125 \%)\end{array}$ & $\begin{array}{c}1 \\
(3.125 \%)\end{array}$ \\
\hline Analgesia & $\begin{array}{c}23 \\
(69.69 \%)\end{array}$ & $\begin{array}{c}5 \\
(15.15 \%)\end{array}$ & $\begin{array}{c}1 \\
(3.03 \%)\end{array}$ & $\begin{array}{c}1 \\
(3.03 \%)\end{array}$ & $\begin{array}{c}1 \\
(3.03 \%)\end{array}$ & $\begin{array}{c}2 \\
(6.06 \%)\end{array}$ \\
\hline $\mathrm{P}=0.836$.
\end{tabular}

Table 3 - Emotional state.

\begin{tabular}{c|c|c|c}
\hline & Calm & Slightly nervous & Very nervous \\
\hline Block & $18(56.25 \%)$ & $12(37.5 \%)$ & $2(6.25 \%)$ \\
\hline Analgesia & $15(45.45 \%)$ & $13(39.39 \%)$ & $5(15.15 \%)$ \\
\hline$P=0.450$
\end{tabular}

Table 4 - Asking whether the type of anesthesia encouraged the patient to undergo the procedure.

\begin{tabular}{c|c|c}
\hline & Yes & No \\
\hline Bloqueio & 0 & $32(100 \%)$ \\
\hline Analgesia & $11(33.33 \%)$ & $22(66.66 \%)$ \\
\hline
\end{tabular}

Table 5 - Asking whether the patient would accept the same type of anesthesia.

\begin{tabular}{c|c|c}
\hline & Yes & No \\
\hline Block & $20(60.5 \%)$ & $12(37.5 \%)$ \\
\hline Analgesia & $33(100 \%)$ & 0 \\
\hline$P=0,000$.
\end{tabular}

\section{DISCUSSION}

Knee arthroscopy has been said to be the orthopedic procedure most performed in the United States. According to the literature, the advantages of performing it under local anesthesia include: lower cost of surgery, possibility of faster recovery and low risk of complications, among others ${ }^{(5,6,10,11)}$.

Even though several studies have shown that this procedure can be successfully carried out by means of local anesthesia, few studies use this type of anesthesia $^{(5)}$, because of the fear that it will be insufficient and will lead to intraoperative pain. This question was the motivation for the present study, in which through comparison with a spinal block method, we sought to assess the quality of local anesthesia with propofol, along with its degree of acceptability among the patients and its impact of an emotional nature on the groups.

In our investigation, we observed that the memory of pain or discomfort during the surgery was low and without difference between the two groups. All the operations were concluded without the need to convert to general anesthesia, thus confirming that the duration of anesthesia was sufficient in all the cases, which corroborates what was observed by Buckley et $a l^{(12)}$.

The results found in the present study have also been observed in other similar studies. In a randomized prospective clinical study comparing spinal anesthesia and local anesthesia using propofol, Dunn et $a l^{(13)}$ observed that $88.9 \%$ of the patients in the LA group subjectively classified their anesthesia as excellent or good, versus 77.4 in the BL group. In another randomized prospective study comparing spinal anesthesia and local anesthesia using propofol, Ben-David et $a l^{(10)}$ found that $90 \%$ of the patients were very satisfied or satisfied in the two groups.

Local anesthesia combined with propofol in the form of continuous infusion was found to provide excellent analgesia, although in isolation, it possibly would not provide a comfortable experience for 
patients, or ideal conditions for the surgery ${ }^{(10)}$. Accompanied by sedation, local anesthesia allowed arthroscopy to be performed without discomfort ${ }^{(14)}$, and the sedation was responsible for the absence of perception of pain ${ }^{(3)}$. Use of local anesthesia alone has been advocated in the literature, but many of these studies recommended that it should only be used in diagnostic procedures ${ }^{(2,6) \text {. }}$

Use of anesthetic in association with a vasoconstrictor is another important factor in carrying out arthroscopy under local anesthesia. According to Atrio et $a l^{(11)}$, use of epinephrine in association with bupivacaine boosts the effect, the duration and the local activity of the anesthetic, through diminishing the absorption velocity and reducing the amount of intra-articular bleeding.

We also assessed the pain reported on the first day after the operation. For this, we used a VAS, a method that has been widely used in the literature ${ }^{(14-17)}$. Our study demonstrated that the amount of pain at this time was similar in the two groups, without any statistical difference, thus showing that the efficacy of postoperative analgesia with local anesthesia using propofol is equivalent to analgesia achieved through spinal block. These findings are compatible with those of Ben-David et al ${ }^{(10)}$, Jacobson ${ }^{(1)}$ and Dunn ${ }^{(13)}$.

However, Jacobson found a significant difference such that a smaller number of patients used analgesics in the group that underwent local anesthesia, compared with the group that underwent spinal anesthesia.

Asking patients whether they would accept the same type of anesthesia after explaining the other options was also used by Barreto et $a l^{(15)}$ to assess the acceptance of the procedure.

We observed in this study that there was significantly greater acceptance of local anesthesia than of spinal block, which coincides with the findings of other studies. Shapiro et $a l^{(5)}$ reported that $84 \%$ of their patients who underwent arthroscopy with local anesthesia would accept the same type of anesthesia. Among the 57 patients of Buckley et $a l^{(12)}$, 93\% would accept the same anesthesia for future arthroscopy. Lintner et al ${ }^{(18)}$ asked their patients about their satisfaction and whether they would accept new surgery with local anesthetic, and found excellent acceptance and satisfaction, with $99 \%$ acquiescence.

A study conducted among children and adolescents who underwent knee arthroscopy under local anes- thesia combined with intravenous propofol showed a high satisfaction rate, such that $93.8 \%$ of the patients would accept undergoing the same anesthesia in a subsequent procedure ${ }^{(19)}$.

In order to assess the patients' emotional state before the surgery, and whether the type of anesthesia influenced their acceptance of the surgery, we asked the patients about their emotional state immediately before the procedure and whether knowing what type of anesthesia was being used encouraged them to undergo the operation. There was no statistically significant difference in the patients' emotional state between the groups. However, we observed that in the LA group, 11 patients (33.3\%) said that they felt encouraged to undergo the surgery after getting to know the type of anesthesia. On the other hand, in the BL group, the anesthesia neither encouraged the patients nor interfered. The analysis on these data revealed that despite the lack of modification of the preoperative anxiety, the use of local anesthesia with sedation may have been a facilitator for patients who had concerns about or aversion to spinal block, and who for such reasons might reject the surgical indication.

We deem it important, whenever possible, to offer patients who were apprehensive about undergoing spinal block the possibility of interacting with the medical team regarding the anesthesia to be used. Shapiro et $a l^{(5)}$ advised their patients about the types of anesthesia and the advantages and disadvantages of each type, and then allowed the patients to make the final decision. They observed that $71.87 \%$ of the patients chose local anesthesia, $23.43 \%$ chose general anesthesia and $4.68 \%$ chose regional or epidural block.

Since knee arthroscopy is generally a minimally invasive procedure that is suitable for day hospitals, there is a need for anesthetic procedures that enable rapid recovery, early walking and sooner yet safe hospital discharge, without occurrences of neurological abnormalities or urine retention. The traditional methods of spinal anesthesia have proven to be problematic for outpatients, because of transitory neurological symptoms ${ }^{(10)}$.

We believe that it is fundamentally important to carefully select patients who are candidates for local anesthesia. Procedures that require open techniques for meniscal suturing or ligament reconstruc- 
tion should not be performed under local anesthesia. Other restrictions also cited in the literature include: patients who are very anxious, acute injuries, surgery on children, very lengthy procedures ${ }^{(20)}$, hypertrophic synovitis, joint stiffness, pain or hip stiffness ${ }^{(21)}$.

Nonetheless, the literature reveals that arthroscopy can be carried out successfully under local anesthesia in $75 \%$ to $92 \%$ of all the procedures in which it is possible to use this technique ${ }^{(5,16)}$.

In conclusion, local anesthesia in association with sedation using propofol provides intraoperative analgesia that is comparable with what anesthetic block provides. It also enables excellent postoperative pain control and allows rapid recovery. Compared with the other protocol that was evaluated, it presents a higher acceptance rate among patients and, despite some restrictions, may serve as a good alternative for spinal block, especially in day hospitals and when restrictions on the use of traditional anesthetic models are imposed by patients.

\section{REFERENCES}

1. Jackson RW. História da artroscopia. In: Andrews JR, Timmerman LA. Artroscopia diagnóstico e cirurgia. Rio de Janeiro: Revinter; 2000. p. 3-5

2. Gomes JLE, Marczyk LRS. Exame artroscópico do joelho sob anestesia local. Rev Bras Ortop. 1982; 17(4):147-50.

3. Barreto JM, Couto P. Artroscopia do joelho sob anestesia local e sedação. Rev Bras Ortop. 1997;32(4):289-92.

4. Eriksson E, Häggmark T, Saartok T, Sebik A, Ortengren B. Knee arthroscopy with local anesthesia in ambulatory patients. Methods, results and patient compliance. Orthopedics. 1986;9(2):186-8.

5. Shapiro MS, Safran MR, Crockett H, Finerman GA. Local anesthesia for knee arthroscopy. Efficacy and cost benefits. Am J Sports Med. 1995;23(1):50-3.

6. Wredmark T, Lundh R. Arthroscopy under local anaesthesia using controlled pressure-irrigation with prilocaine. J Bone Joint Surg Br. 1982;64(5):583-5.

7. Fairclough JA, Graham GP, Pemberton D. Local or general anaesthetic in day case arthroscopy? Ann R Coll Surg Engl. 1990;72(2):104-7.

8. Horlocker TT, Hebl JR. Anesthesia for outpatient knee arthroscopy: is there na optimal technique? Reg Anesth Pain Med. 2003;28(1):58-63.

9. Forssblad M, Weidenhielm L. Knee arthroscopy in local versus general anaesthesia. The incidence of rearthroscopy. Knee Surg Sports Traumatol Arthrosc. 1999;7(5):323-6.

10. Ben-David B, DeMeo PJ, Lucyk C, Solosko D. A comparison of minidose lidocaine-fentanyl spinal anesthesia and local anesthesia/propofol infusion for outpatient knee arthroscopy. Anesth Analg. 2001;93(2):319-25.

11. Atrio GAP, Roman FA, Garcia JT. Bupivacaína como anestésico loca em las artroscopias de rodilla. Rev cubana Ortop Traumatol. 1999; 13(1-2): $27-30$
12. Buckley JR, Hood GM, Macrae W. Arthroscopy under local anaesthesia. J Bone Joint Surg Br. 1989;71(1):126-7.

13. Dunn WR, Cordasco FA, Flynn E, Jules K, Gordon M, Liguori G. A prospective randomized comparison of spinal versus local anesthesia with propofol infusion for knee arthroscopy. Arthroscopy. 2006;22(5):479-83.

14. Arai YC, Ikeuchi M, Fukunaga K, Ueda W, Kimura T, Komatsu T. Intra-articular injection of warmed lidocaine improves intraoperative anaesthetic and postoperative analgesic conditions. Br J Anaesth. 2006;96(2):259-61.

15. Barreto JM, Victória CH, Bedoya FT. Artroscopia do joelho com anestesia local e sedação: estudo comparativo entre três protocolos de sedação venosa utilizando sistema análogo-visual. Rev Bras Ortop. 1992; 27(6): 383-386.

16. Jacobson E, Forssblad M, Rosenberg J, Westman L, Weidenhielm L. Can local anesthesia be recommended for routine use in elective knee arthroscopy? A comparison between local, spinal, and general anesthesia. Arthroscopy. 2000;16(2):183-90.

17. Takahashi T, Tanaka M, Ikeuchi M, Sadahiro T, Tani T. Pain in arthroscopic knee surgery under local anesthesia. Acta Orthop Scand. 2004;75(5):580-3.

18. Lintner S, Shawen S, Lohnes J, Levy A, Garrett W. Local anesthesia in outpatient knee arthroscopy: a comparison of efficacy and cost. Arthroscopy. 1996;12(4):482-8

19. Maldini B, Miskulin M. Outpatient arthroscopic knee surgery under combined local and intravenous propofol anesthesia in children and adolescents. Paediatr Anaesth. 2006;16(11):1125-32.

20. Williams CR, Thomas NP. A prospective trial of local versus general anaesthesia for arthroscopic surgery of the knee. Ann R Coll Surg Engl. 1997;79(5):345-8.

21. Law BK, Yung PS, Ho EP, Chang JJ, Kwok GK, Fung KY, Chan KM. Review of knee arthroscopy performed under local anesthesia. Sports Med Arthrosc Rehabil Ther Technol. 2009;1(1):3. 